list of available reports is often published in the Psychiatric Bulletin and can be obtained from the College.

Chris Thompson, Registrar, The Royal College of Psychiatrists
This is one of a series of articles about the way the College works. The Registrar would be interested to recetve suggestions and comments from Members, Fellows and trainees

\title{
New Council Report
}

\section{Strategies for the Management of Dis- turbed and Violent Patients in Psy- chiatric Units}

This is a joint review by the Royal College of Psychiatrists and the Royal College of Nursing of their policies for the overall management of violence and aggression in hospitalised patients. It stemmed from the widespread and wellfounded concern about the potential for the misuse of selection and examines alternative approaches.

The emphasis of the report is on the prevention of violence with a reduction in aggression. There is an account of a therapeutic regimen which might serve as a model for in-patient units. This regimen demands a substantial investment in the ward environment as well as in staff training, support and supervision, as much will depend on the attitudes of staff to violence and its management. Also considered is the part to be played by different professions in a multidisciplinary approach. A central element is the assessment of risk as is the involvement of the patient in the strategy of his/her treatment.

Although often misused, medication has an important and useful place in treatment, but must be subject to frequent and careful review.

Restraint should be subject to the formal framework of 'control and restraint' techniques which require supervised training and monitoring. The nature and place of restraint requires further scrutiny.
Seclusion is an emergency measure to be used only where there is a significant risk of harm to the patient or others. It should be used as infrequently as possible and only for as long as it takes for the patient to return to a calmer frame of mind. In short, it should be a short-lived 'fireextinguisher' and should not form part of any therapeutic programme. Although it was suggested that it be phased out altogether, there remain some occasions when alternatives are inadequate or more unpleasant. Furthermore, its removal should carry the risk that the practice would continue but under another name: seclusion has the advantage that its use is controlled. recorded and externally monitored.

Indeed, any form of emergency response should be recorded systematically, subject to audit, and be brought to the attention of the Unit's managers and purchasers. The appendices include various sample reporting forms.

Also appended is a consensus protocol for a broader approach to the management of aggression. including out-patients, which was prepared by the Section for the Psychiatry of Learning Disability. Finally there is a comprehensive bibliography.

The full report (please quote CR41) is available from the College Publications Department (Kerstin Sayoud, ext. 146) at a cost of $\$ 5$ per copy. 